\title{
Wiener Polarity Index of Cycle-Block Graphs
}

\author{
Zhen Jia, Fuyi Wei, and Yang Wu \\ Department of Mathematics, South China Agricultural University, Guangzhou 510642, China \\ Correspondence should be addressed to Fuyi Wei; weifuyi@scau.edu.cn
}

Received 18 August 2013; Accepted 6 January 2014; Published 27 February 2014

Academic Editors: X. M. Yang and G. Yu

Copyright (C) 2014 Zhen Jia et al. This is an open access article distributed under the Creative Commons Attribution License, which permits unrestricted use, distribution, and reproduction in any medium, provided the original work is properly cited.

The Wiener polarity index $W_{P}$ of a graph $G$ is the number of unordered pairs of vertices $u, v$ of $G$ such that the distance $d_{G}(u, v)$ between $u$ and $v$ is 3 . Cycle-block graph is a connected graph in which every block is a cycle. In this paper, we determine the maximum and minimum Wiener polarity index of cycle-block graphs and describe their extremal graphs; the extremal graphs of 4-uniform cactus with respect to Wiener polarity index are also discussed.

\section{Introduction}

Let $G=(V, E)$ be a connected simple graph. The distance $d_{G}(u, v)$ between the vertices $u$ and $v$ of $G$ is defined as the length of a shortest path connecting $u$ and $v \cdot N_{G}^{i}(u)=\{v \in$ $\left.V(G) \mid d_{G}(u, v)=i\right\}$ is called the $i$ th neighbor set of $u . d(u)=$ $\left|N_{G}^{1}(u)\right|$ is called the degree of $u$. If $d(u)=1$, then $u$ is called a pendant vertex of $G$. $C_{l}=u_{1} u_{2} \cdots u_{l} u_{1}$ denotes a cycle of $\operatorname{order} l(\geq 3)$. The girth of $G$, denoted by $g$, is the length of the shortest cycle of $G$.

A block of the graph $G$ is a maximal 2-connected subgraph of $G$. A cactus graph is a connected graph in which no edge lies in more than one cycle, such that each block of a cactus graph is either an edge or a cycle. If all blocks of a cactus $G$ are cycles, the graph is defined as cycle-block graph. In this paper, suppose the cycle-block graph consist of $n$ cycles, the length of the cycles may be different. If all blocks of a cactus $G$ are cycles of the same length $m$, the cactus is $m$-uniform. A hexagonal cactus is a 6-uniform cactus that every block of the graph is a hexagon. A vertex shared by two or more hexagons is called a cut-vertex. If each hexagon of a hexagonal cactus $G$ has at most two cut-vertices and each cut-vertex is shared by exactly two hexagons, we say that $G$ is a chain hexagonal cactus (see Figure 1(a)). A star cactus is a cactus consisting of $n$ cycles, spliced together in a single vertex $u$ (see Figure 1(b)). A star hexagonal cactus is a star cactus in which every cycle is a hexagon.

The Wiener polarity index of $G$, denoted by $W_{P}(G)$, is the number of unordered vertex pairs of distance 3 . It was first used in a linear formula to calculate the boiling points $t_{B}$ of paraffin [1]: of paraffin:

$$
t_{B}=a W(G)+b W_{P}(G)+c
$$

where $a, b, c$ are constants for a given isomeric group.

The Wiener polarity index became popular recently, and many mathematical properties and its chemical applications were discovered [2-6]. In this line, Du et al. [2] characterized the minimum and maximum Wiener polarity index among all trees of order $n$, and Deng [3] determined the largest Wiener polarity indices among all chemical trees of order $n$. M. H. Liu and B. L. Liu [4] determined the first two smallest Wiener polarity indices among all unicyclic graphs of order $n$. Hou et al. [5] determined the maximum Wiener polarity index of unicyclic graphs. Behmarama et al. [6] computed the Wiener polarity index of hexagonal cacti. To know more about cactus graph one can research $[7,8]$.

In this paper, we discuss the extremal graphs of Wiener polarity index of cycle-block graphs with $g \geq 5$ and 4-uniform cactus.

\section{The Extremal Graphs of Wiener Polarity Index of Cycle-Block Graphs with $g \geq 5$}

In this section, we characterize the maximum and minimum Wiener polarity index of the cycle-block graphs with $g \geq 5$.

Suppose that $G_{1}$ and $G_{2}$ are two connected graphs. The graph obtained by identifying a chosen vertex of $G_{1}$ and 


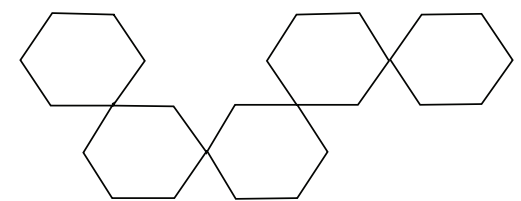

(a)

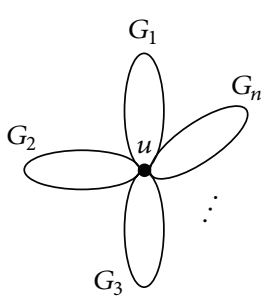

(b)

Figure 1: A chain hexagonal cactus (a) and a star cactus graph (b).

another of $G_{2}$ is called the coalescence of $G_{1}$ and $G_{2}$, denoted by $G_{1} \circ G_{2}$. The vertex identifying $G_{1}$ and $G_{2}$ is called the coalescence vertex. The cycle-block graph which consist of $n$ cycles $C_{1}, C_{2}, \ldots, C_{n}$ can be seen as the coalescence of cycles by $n-1$ times, $G \cong C_{1} \circ C_{2} \circ \cdots \circ C_{n}$.

Lemma 1. Let $G_{1}$ and $G_{2}$ be two connected graphs; suppose that $v_{0}$ is the coalescence vertex of $G_{1} \circ G_{2}$. Then $W_{P}\left(G_{1} \circ G_{2}\right)=$ $W_{P}\left(G_{1}\right)+W_{P}\left(G_{2}\right)+\sum_{k=1}^{2}\left|N_{G_{1}}^{k}\left(v_{0}\right) \| N_{G_{2}}^{3-k}\left(v_{0}\right)\right|$.

Proof. Suppose that $u, v \in V\left(G_{1} \circ G_{2}\right)$ and $d_{G_{1} \circ G_{2}}(u, v)=3$. Then,

$$
\begin{gathered}
\left|\left\{(u, v) \mid d_{G_{1} \circ G_{2}}(u, v)=3, u, v \in V\left(G_{i}\right)\right\}\right|=W_{P}\left(G_{i}\right), \\
i=1,2, \\
\left|\left\{(u, v) \mid d_{G_{1} \circ G_{2}}(u, v)=3, u \in V\left(G_{1}\right), v \in V\left(G_{2}\right)\right\}\right| \\
=\sum_{k=1}^{2}\left|N_{G_{1}}^{k}\left(v_{0}\right)\right|\left|N_{G_{2}}^{3-k}\left(v_{0}\right)\right| .
\end{gathered}
$$

By the definition of Wiener polarity index, the result holds.

Theorem 2. Let $G$ be a cycle-block graph with $g \geq 5$ and let $G$ be consisting of $n$ cycles $C_{1}, C_{2}, \ldots, C_{n}$. Then

$$
W_{P}(G) \leq \sum_{i=1}^{n} W_{P}\left(C_{i}\right)+8\left(\begin{array}{c}
n \\
2
\end{array}\right)
$$

the equality holds if and only if $G \cong G^{*}$, where $G^{*}$ is a star cactus graph.

Proof. Let $G$ be a cycle-block graph with $g \geq 5$. Here we apply induction to $n$.

When $k=2$, the graph is determined uniquely. By Lemma 1 and elementary computation, we have $W_{P}(G)=$ $\sum_{i=1}^{2} W_{P}\left(C_{i}\right)+8$.

When $k=3$, there are three cycles $C_{1}, C_{2}$, and $C_{3}$. The graph $G$ is obtained from $C_{1} \circ C_{2}$ by attaching a new cycle $C_{3}$ to $C_{1} \circ C_{2}$. There are three ways to attach the cycle $C_{3}$. Here we suppose that $v_{0}$ is the coalescence vertex of $C_{1} \circ C_{2}$ and $v_{1}$ is the new coalescence vertex of $C_{1} \circ C_{2} \circ C_{3}$.
By Lemma 1 and elementary computation, we have

$$
W_{P}(G)= \begin{cases}\sum_{i=1}^{3} W_{P}\left(C_{i}\right)+8\left(\begin{array}{l}
3 \\
2
\end{array}\right), & d\left(v_{0}, v_{1}\right)=0, \\
\sum_{i=1}^{3} W_{P}\left(C_{i}\right)+20, & d\left(v_{0}, v_{1}\right)=1, \\
\sum_{i=1}^{3} W_{P}\left(C_{i}\right)+16, & d\left(v_{0}, v_{1}\right) \geq 2,\end{cases}
$$

where $d\left(v_{0}, v_{1}\right)=0$ implies that $G$ is the star cactus graph. From the result of the three cases, it holds for the result.

Now suppose that the assertion holds for $k=n-1$. Next we prove that the result holds for $k=n$.

Suppose that $v_{0}$ is the coalescence vertex of $C_{1} \circ C_{2} \circ \ldots \circ$ $C_{n-1}$ and $v_{1}$ is the coalescence vertex of $C_{1} \circ C_{2} \circ \cdots \circ C_{n}$. Three cases occur

$$
W_{P}(G)= \begin{cases}\sum_{i=1}^{n} W_{P}\left(C_{i}\right)+8\left(\begin{array}{c}
n \\
2
\end{array}\right), & d\left(v_{0}, v_{1}\right)=0, \\
\sum_{i=1}^{n} W_{P}\left(C_{i}\right)+8\left(\begin{array}{c}
n-1 \\
2
\end{array}\right)+4 n, & d\left(v_{0}, v_{1}\right)=1, \\
\sum_{i=1}^{n} W_{P}\left(C_{i}\right)+8\left(\begin{array}{c}
n-1 \\
2
\end{array}\right)+8, & d\left(v_{0}, v_{1}\right) \geq 2,\end{cases}
$$

where $d\left(v_{0}, v_{1}\right)=0$; it implies that $G$ is the star cactus graph. Obviously, the star cactus graph has the maximum Wiener polarity index of cycle-block graphs with $g \geq 5$. This completes the proof.

Let $\mathbb{G}$ be a graph set that consists of the cycle-block graphs of $n$ cycles with $n-1$ cut-vertices and the distance between any cut-vertices being greater than 1 . By a similar method with Theorem 2, we have Theorem 3; the proof is in the Appendix.

Theorem 3. Let $G$ be a cycle-block graph with $g \geq 5$ and let $G$ be consisting of $n$ cycles $C_{1}, C_{2}, \ldots, C_{n}$. Then

$$
W_{P}(G) \geq \sum_{i=1}^{n} W_{P}\left(C_{i}\right)+8(n-1) ;
$$

the equality holds if and only if $G \in \mathbb{G}$.

Remark 4. By Theorems 2 and 3, we know that the cycle-block graph with maximum Wiener polarity index is determined 


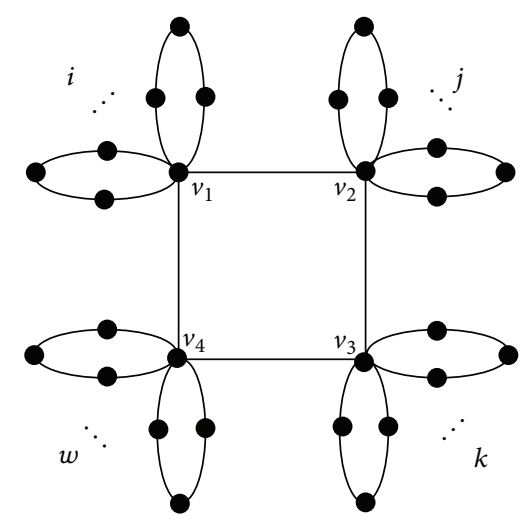

FIGURE 2: The graph of $Q_{n}(i, j, k, \omega)$.

uniquely while the cycle-block graph with minimum Wiener polarity index is not.

For some types of hexagonal cacti which represent common chemical structures, as an extension, we obtain the 6uniform cactus with the maximum and minimum Wiener polarity index.

Corollary 5. Let $G$ be 6-uniform cactus with $n$ hexagons. Then $11 n-8 \leq W_{P}(G) \leq 4 n^{2}-n$; the first equality holds if and only if there are $n-1$ different cut-vertices in $G$ and distance between any two vertices is greater than 1; the second equality holds if and only if $G$ is a star hexagonal cactus graph.

\section{The Extremal Graphs of Wiener Polarity Index of 4-Uniform Cactus}

The case of 4-uniform cactus' extremal graphs is different from the cycle-block graphs with $g \geq 5$ and more complex. Let $Q_{n}(i, j, t, w)$ be a graph obtained from a quadrilateral $C_{4}=v_{1} v_{2} v_{3} v_{4} v_{1}$ by attaching $i, j, t$, and $w$ quadrilaterals to vertices $v_{1}, v_{2}, v_{3}$, and $v_{4}$, respectively, where $i+j+t+w+1=n$ (see Figure 2).

Theorem 6. Suppose that $G$ is 4-uniform withn ( $\geq 2)$ quadrilaterals. Then

$$
W_{P}(G) \leq 2 n^{2}-2 n
$$

the equality holds if and only if $G \cong Q_{n}(i, j, 0,0)$, where $i \geq$ $0, j=n-1-i$.

Proof. We apply induction to $n$.

When $k=2, G$ is unique. By Lemma 1 , we have $W_{P}(G)=$ 4 ; the result follows.

When $k=3$, we have

$$
W_{P}(G)= \begin{cases}12, & G \cong Q_{3}(2,0,0,0), \\ 12, & G \cong Q_{3}(1,1,0,0), \\ 8, & G \cong Q_{3}(1,0,1,0) ;\end{cases}
$$

the result holds.

Suppose that the result holds for $k=n-1$. When $k=n$, there is a new quadrilateral to be attached to $Q_{n-1}(i, j, 0,0)$, where $j=n-2-i, i \geq 0$. Without loss of generality, suppose that $i>0$; there are two cases to be discussed concerning of the variable parameters of $j$.

Case $1(j=0)$. It is a star quadrilateral cactus. Three new graphs $Q_{n}(n-1,0,0,0), Q_{n}(n-2,1,0,0)$, and $Q_{n}(n-2,0,1,0)$ are obtained by three different ways to attach a quadrilateral to $Q_{n-1}(n-2,0,0,0)$.

By elementary computation and Lemma 1, we have

$$
\begin{aligned}
& W_{P}(G)=\left\{\begin{array}{l}
2(n-1)^{2}-2(n-1) \\
\quad+2 \times(n-1)+1 \times 2(n-1), \\
2(n-1)^{2}-2(n-1) \\
\quad+2 \times(2(n-2)+1)+1 \times 2, \\
2(n-1)^{2}-2(n-1) \\
+2 \times 1+1 \times 2,
\end{array}\right. \\
&=\left\{\begin{array}{cc}
2 n^{2}-2 n, & G \cong Q_{n}(n-1,0,0,0), \\
2 n^{2}-2 n, & G \cong Q_{n}(n-2,1,0,0), \\
2 n^{2}-6 n+8, & G \cong Q_{n}(n-2,0,1,0) .
\end{array}\right.
\end{aligned}
$$

For these three subcases, the result follows.

Case $2(j>0)$. There are also three subcases to attach a new quadrilateral to $Q_{n-1}(i, j, 0,0)$.

Subcase $1 . G \cong Q_{n}(i+1, j, 0,0)$ or $G \cong Q_{n}(i, j+1,0,0)$.

We only prove the case $G \cong Q_{n}(i+1, j, 0,0) . G \cong Q_{n}(i, j+$ $1,0,0)$ can be proved similarly. By elementary computation and Lemma 1, we have

$$
\begin{aligned}
W_{P}\left(Q_{n}(i+1, j, 0,0)\right)= & 2(n-1)^{2}-2(n-1) \\
& +2 \times(i+1+2 j)+1 \times 2(i+1) \\
= & 2 n^{2}-2 n .
\end{aligned}
$$

Subcase 2. $G \cong Q_{n}(i, j, 1,0)$ or $G \cong Q_{n}(i, j, 0,1)$.

When $G \cong Q_{n}(i, j, 1,0)$, by elementary computation and Lemma 1, we have

$$
\begin{aligned}
W_{P}\left(Q_{n}(i, j, 1,0)\right)= & 2(n-1)^{2}-2(n-1)+2 \times(2 j+1) \\
& +1 \times 2 \\
= & 2 n^{2}-6 n+4 j+8 .
\end{aligned}
$$

Similarly, $W_{P}\left(Q_{n}(i, j, 0,1)\right)=2 n^{2}-6 n+4 i+8$.

Subcase 3. The new quadrilateral attach to one of the $i$ or $j$ quadrilaterals and create a new cut-vertex different from $v_{1}$ and $v_{2}$.

When a new quadrilateral is attached to one of the $j$ quadrilaterals. The distance between the new cut-vertex and its nearest cut-vertex is 1 . By elementary computation and Lemma 1, we have

$$
\begin{aligned}
W_{P}(G) & =2(n-1)^{2}-2(n-1)+2 \times(1+2 j)+1 \times 2 \\
& =2 n^{2}-6 n+4 j+8 .
\end{aligned}
$$




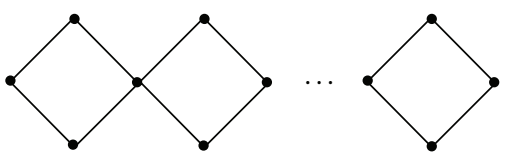

FIgURE 3: The graph of $G^{\prime}$.

Analogously, when the new quadrilateral is attached to one of the $i$ quadrilaterals, we have $W_{P}(G)=2 n^{2}-6 n+4 i+8$.

When the distance between the new cut-vertex and its nearest cut-vertex is 2. By elementary computation and Lemma 1, we have

$$
\begin{aligned}
W_{P}(G) & =2(n-1)^{2}-2(n-1)+2 \times 1+1 \times 2 \\
& =2 n^{2}-6 n+8 .
\end{aligned}
$$

By the discussion of Case 2 above, for $i>0, j>0$, it is easy to verify that $2 n^{2}-2 n>\max \left\{2 n^{2}-6 n+4 i+12,2 n^{2}-6 n+\right.$ $\left.4 j+12,2 n^{2}-6 n+8\right\}$. This completes the proof.

With the analogous method with Theorem 3, we can deduce the minimum Wiener polarity index of 4-uniform (see the proof of the Appendix).

Theorem 7. Let $G$ be 4-uniform with $n$ quadrilaterals. Then

$$
W_{P}(G) \geq 4(n-1) ;
$$

the equality holds if and only if $G \cong G^{\prime}$, where $G^{\prime}$ is a chain quadrilateral cactus with the distance between the cut-vertices being at least 2 (see Figure 3).

Remark 8. From Theorems 6 and 7, we can conclude that the extremal 4-uniform with the maximum Wiener polarity index is not unique, but the minimum case is unique and different from the case of cycle-block graphs with $g \geq 5$. For the case of extremal cycle-block with $g \geq 3$ of Wiener polarity index is very complex, we do not discuss it here.

\section{Appendix}

The proof of Theorem 3 .

Proof. We proof the result with the similar method of Theorem 2.

When $k=2$, the graph is $C_{1} \circ C_{2}$. By Lemma 1 and elementary computation, we have $W_{P}(G)=\sum_{i=1}^{2} W_{P}\left(C_{i}\right)+8$.

When $k=3$, from the process of the proof of Theorem 2 , Case 3 has the minimum Wiener polarity index. It holds for the result.

Suppose that $k=n-1$ holds for the result; the smallest Wiener polarity index of cycle-block graphs is the cycleblock graph with $n-2$ cut-vertex and any two cut-vertices' distance being greater than 1 . Next we demonstrate that the result holds for $k=n$. There are four cases to be discussed. Suppose that $v_{1}, v_{2}, \ldots, v_{n-2}$ are cut-vertices between the cycles $C_{1}, C_{2}, \ldots, C_{n-1} \cdot v_{n-1}$ is the cut-vertex of $C_{1} \circ C_{2} \circ$ $\cdots \circ C_{n}$. Consider the following:

$$
\begin{aligned}
& W_{P}(G)=\left\{\begin{array}{l}
\sum_{i=1}^{n-1} W_{P}\left(C_{i}\right)+8(n-2) \\
+W_{P}\left(C_{n}\right)+2 \times 4+2 \times 4, \\
\sum_{i=1}^{n-1} W_{P}\left(C_{i}\right)+8(n-2) \\
+W_{P}\left(C_{n}\right)+2 \times 6+2 \times 2, \\
\sum_{i=1}^{n-1} W_{P}\left(C_{i}\right)+8(n-2) \\
+W_{P}\left(C_{n}\right)+2 \times 4+2 \times 2, \\
\sum_{i=1}^{n-1} W_{P}\left(C_{i}\right)+8(n-2) \\
+W_{P}\left(C_{n}\right)+2 \times 2+2 \times 2,
\end{array}\right. \\
& \begin{cases}\sum_{i=1}^{n} W_{P}\left(C_{i}\right)+8 n, & d\left(v_{i}, v_{n-1}\right)=0, \\
\sum_{i=1}^{n} W_{P}\left(C_{i}\right)+8 n, & 1 \leq i \leq n-2, \\
& d\left(v_{i}, v_{n-1}\right) \\
& =d\left(v_{j}, v_{n-1}\right)=1, \\
\sum_{i=1}^{n} W_{P}\left(C_{i}\right)+8 n-4, & 1 \leq i, j \leq n-2, i \neq j, \\
& d\left(v_{j}, v_{n-1}\right)=1, \\
& d\left(v_{i}, v_{n-1}\right)>1, \\
& 1 \leq i, j \leq n-2, i \neq j, \\
\sum_{i=1}^{n} W_{P}\left(C_{i}\right)+8(n-1), & d\left(v_{i}, v_{n-1}\right) \geq 2, \\
& 1 \leq i \leq n-2 .\end{cases}
\end{aligned}
$$

From the discussion for $k=n$, we find that the result follows. By induction, it follows that the cycle-block graph $G \in \mathbb{G}$ has the minimum Wiener polarity index.

The proof of Theorem 7 .

Proof. We prove the result with the same method of Theorem 3.

When $k=2$ and $k=3$, from the process of the proof of Theorem 6, it holds for the result.

Suppose that $k=n-1$ holds for the result; next we prove the case of $k=n$ quadrilaterals. When $k=n$, it is the graph that by attaching a new quadrilateral to the chain quadrilateral cactus of $n-1$ quadrilaterals which the distance between the cut-vertices is at least 2 . There are four cases to be discussed. Suppose that $v_{1}, v_{2}, \ldots, v_{n-2}$ are cut-vertices of the chain quadrilateral cactus with $n-1$ quadrilaterals. $v_{n-1}$ is the new coalescence vertex of $C_{1} \circ C_{2} \circ \cdots \circ C_{n}$. Consider the following:

$$
W_{P}(G)=\left\{\begin{array}{l}
4(n-2)+2 \times 2+1 \times 4, \\
4(n-2)+2 \times 5+1 \times 2, \\
4(n-2)+2 \times 3+1 \times 2, \\
4(n-2)+2 \times 1+1 \times 2,
\end{array}\right.
$$




$$
=\left\{\begin{array}{rrr}
4 n, & d\left(v_{i}, v_{n-1}\right)=0, \\
1 \leq i \leq n-2, & \\
4 n+4, & d\left(v_{i}, v_{n-1}\right)=d\left(v_{j}, v_{n-1}\right)=1, \\
1 \leq i, j \leq n-2, i \neq j, & \\
4 n, & d\left(v_{j}, v_{n-1}\right)=1, d\left(v_{i}, v_{n-1}\right)>1, \\
& 1 \leq i, \quad j \leq n-2, \quad i \neq j, \\
4(n-1), & d\left(v_{i}, v_{n-1}\right) \geq 2, \\
& 1 \leq i \leq n-2 .
\end{array}\right.
$$

When $d\left(v_{i}, v_{n-1}\right) \geq 2,1 \leq i \leq n-2$, graph $G$ is the chain quadrilateral cactus of $n-1$ quadrilaterals with the distance between the cut-vertices being at least 2; the result follows. By induction, it follows that the chain quadrilateral cactus with the distance between the cut-vertices being at least 2 has the minimum Wiener polarity index. This completes the proof.

\section{Conflict of Interests}

The authors declare that there is no conflict of interests regarding the publication of this paper.

\section{Acknowledgments}

The authors are grateful to the referees for the helpful comments and useful suggestions on this paper. This work is supported by National Natural Science Foundation of China (nos. 11071088, 11201156).

\section{References}

[1] H. Wiener, "Structural determination of paraffin boiling points," Journal of the American Chemical Society, vol. 69, no. 1, pp. 1720, 1947.

[2] W. Du, X. Li, and Y. Shi, "Algorithms and extremal problem on Wiener polarity index," MATCH: Communications in Mathematical and in Computer Chemistry, vol. 62, no. 1, pp. 235-244, 2009.

[3] H. Deng, "On the extremal Wiener polarity index of chemical trees," MATCH: Communications in Mathematical and in Computer Chemistry, vol. 66, no. 1, pp. 305-314, 2011.

[4] M. H. Liu and B. L. Liu, "On the Wiener polarity index," MATCH: Communications in Mathematical and in Computer Chemistry, vol. 66, no. 1, pp. 293-304, 2011.

[5] H. Q. Hou, B. L. Liu, and Y. F. Huang, "The maximum Wiener polarity index of unicyclic graphs," Applied Mathematics and Computation, vol. 218, pp. 10149-10157, 2012.

[6] A. Behmarama, H. Yousefi-Azaria, and A. R. Ashafi, "Wiener polarity index of fullerenes and hexagonal systems," Applied Mathematics Letters, vol. 25, no. 10, pp. 1510-1513, 2012.

[7] T. Došlić and F. Måløy, "Chain hexagonal cacti: matchings and independent sets," Discrete Mathematics, vol. 310, no. 12, pp. 1676-1690, 2010.

[8] T. Došlić and M. S. Litz, "Matchings and independent sets in polyphenylene chains," MATCH: Communications in Mathematical and in Computer Chemistry, vol. 67, no. 2, pp. 313-330, 2012. 


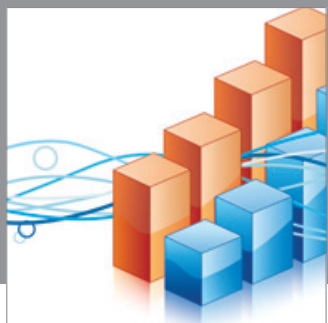

Advances in

Operations Research

mansans

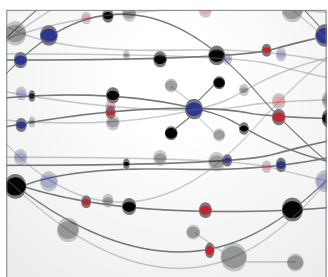

The Scientific World Journal
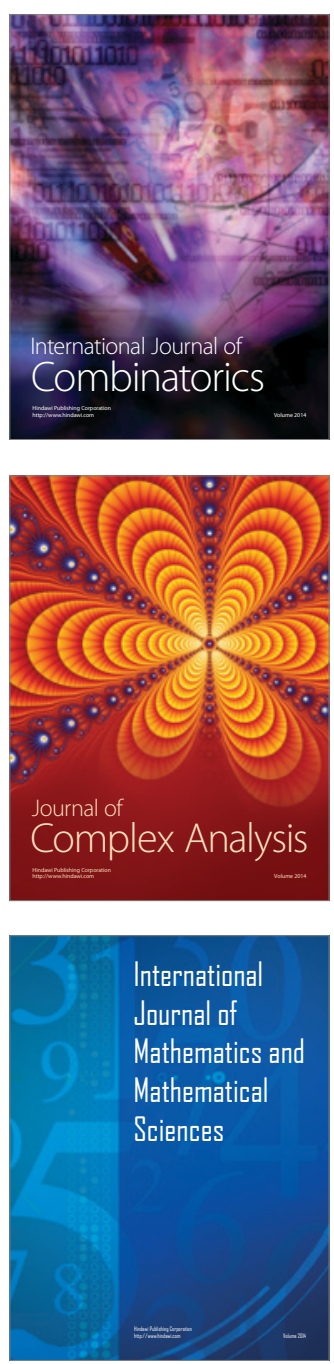
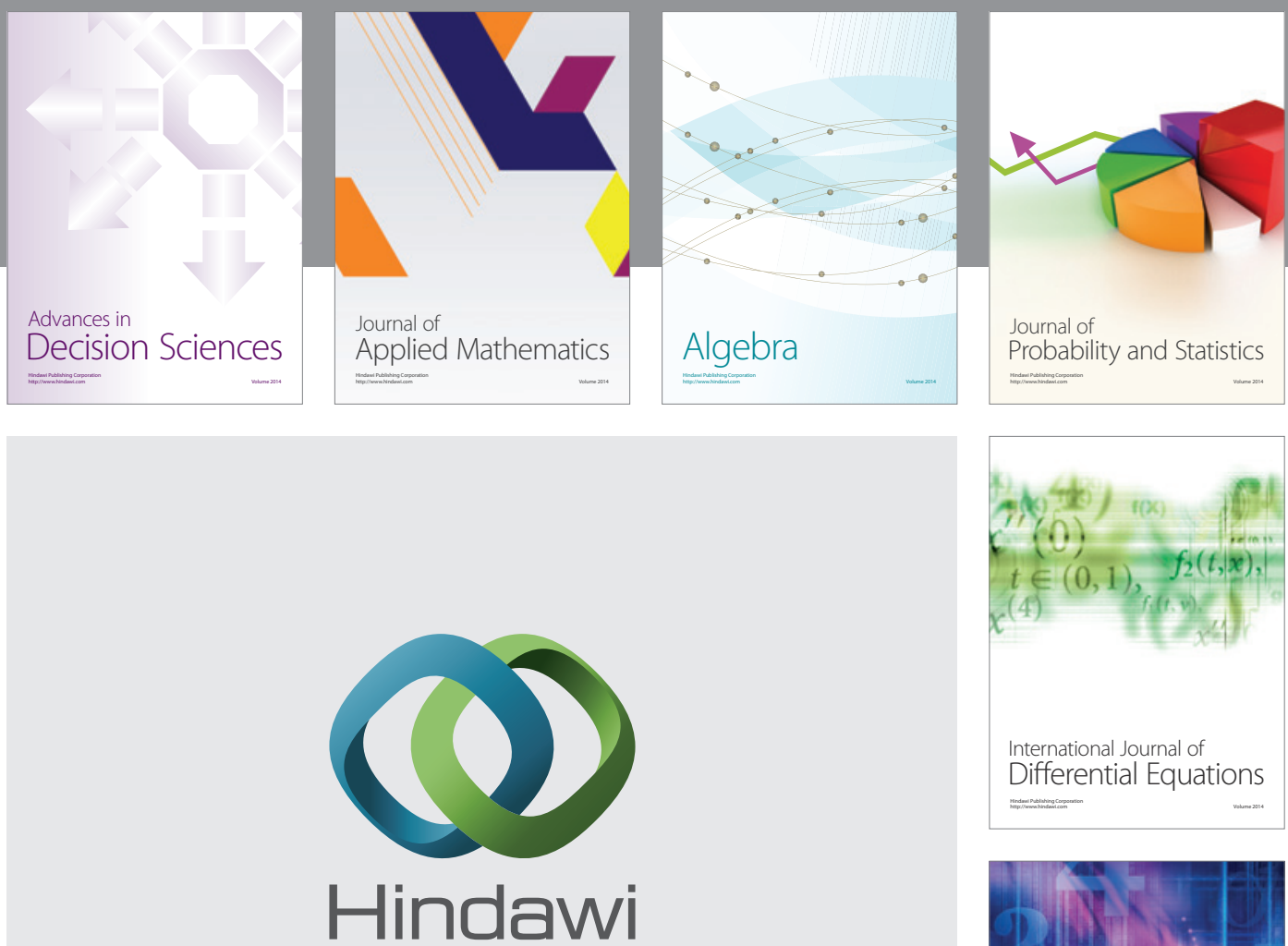

Submit your manuscripts at http://www.hindawi.com
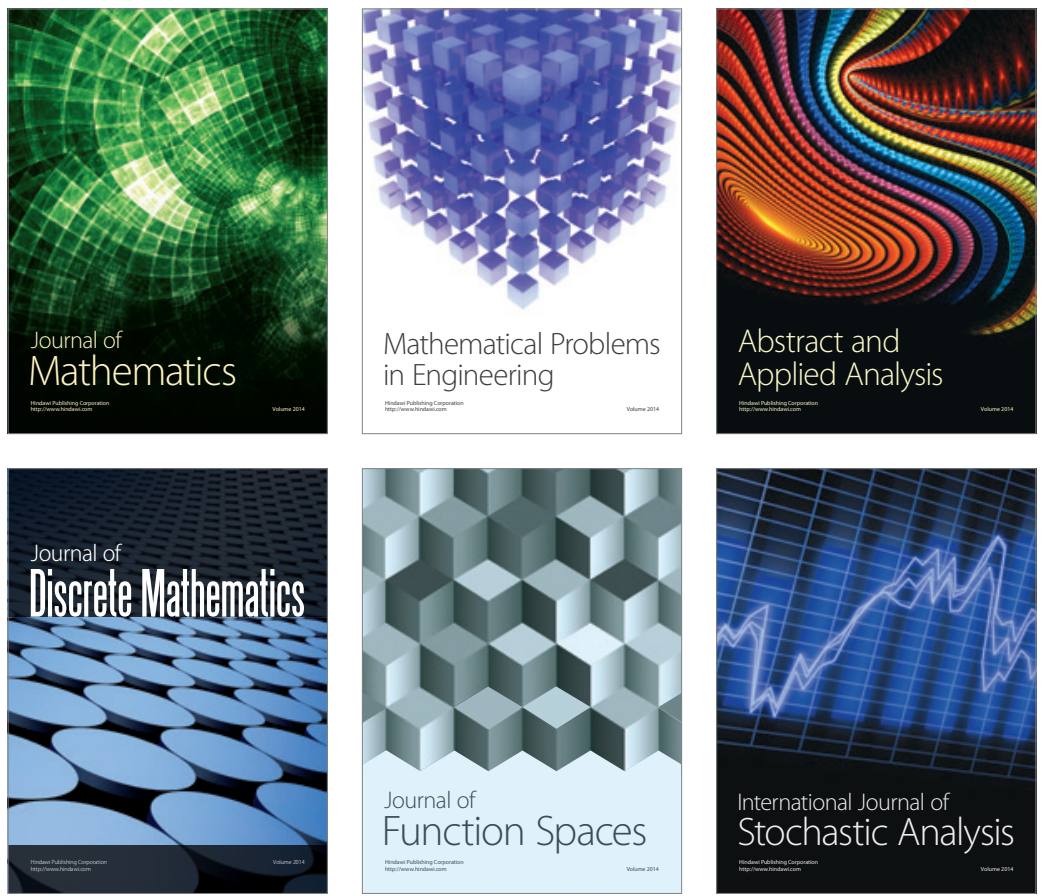

Journal of

Function Spaces

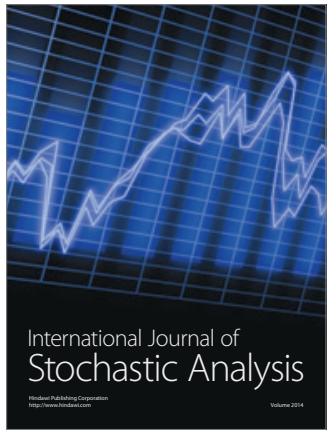

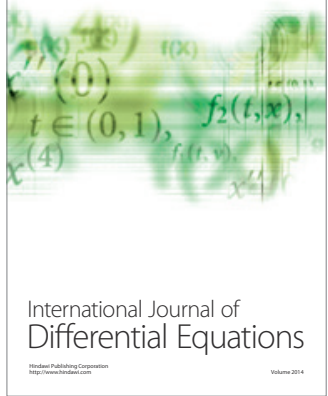
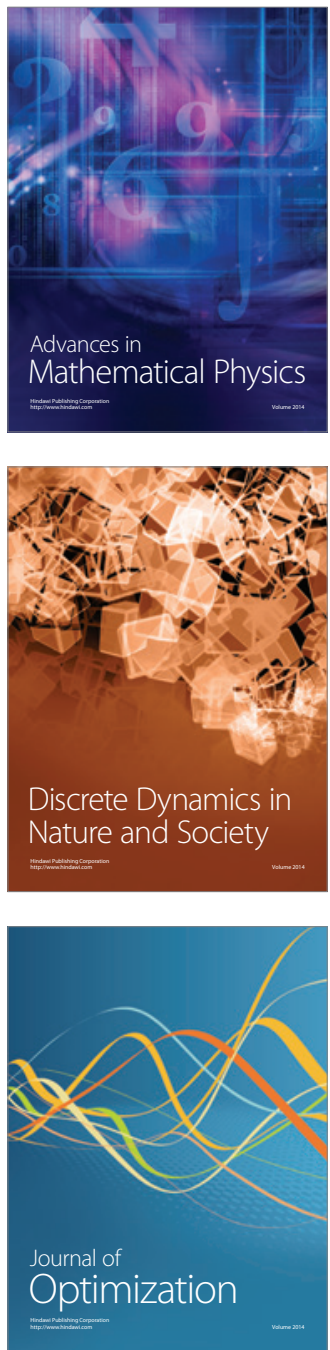